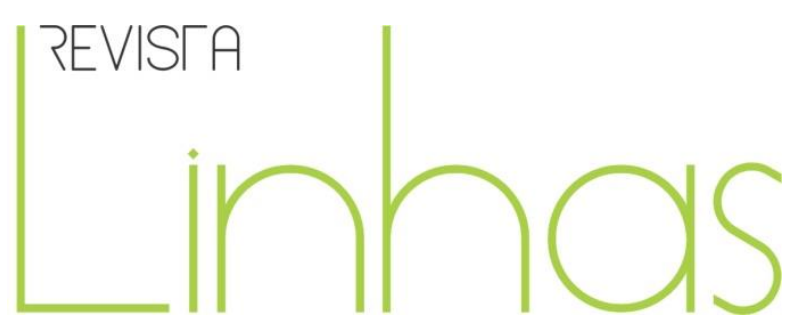

\title{
Curricolo zero-sei: conoscenze fatte di scoperte e riflessioni
}

\begin{abstract}
Riassunto
L'articolo prende in esame la Legge 107/2015 e il successivo Decreto legislativo n.65 del 2017 per analizzare e discutere le implicazioni pedagogiche connesse all'istituzione del "Sistema integrato di educazione e istruzione dalla nascita ai sei anni", prospettiva a partire dalla quale il panorama dei servizi per l'infanzia italiani dovranno confrontarsi per elaborare idee, proposte, pratiche educative relative al curricolo zero-sei. Il quadro legislativo e le acquisizioni del mondo della ricerca sull'infanzia convergono nel delineare l'esigenza di operare a partire da un approccio olistico allo sviluppo nei primi anni di vita e in sintonia con questa visione, anche la proposta di un curricolo zero-sei chiede di essere interpretata e declinata in chiave olistica, connettendo arte, scienze, linguaggi oltre ogni distinzione disciplinare. Le riflessioni proposte da questo scritto prendono in esame allora le specificità dei percorsi di scoperta e di conoscenza propri di alcune discipline, mostrando come la divisione settoriale, a cui un'eccessiva "secondarizzazione" degli apprendimenti ci aveva abituato, sia un elemento estremamente distante dalla pratica degli stessi ricercatori e studiosi. Il procedere per tentativi, sperimentando percorsi anche dissonanti, lontani dalla ricerca di un solo risultato, forzatamente identico per tutti, è dunque una modalità qui suggerita, recuperando e valorizzando esperienze realizzate negli anni proprio nei curricoli pensati per i più piccoli.
\end{abstract}

Parole chiave: Servizi Educativi e Scolastici per L'Infanzia, Continuità Educativa. Arte. Scienza. Scoperta. Esplorazione.
Franca Giuliana Zuccoli

Università degli Stud di Milano-

Bicocca - Italia

franca.zuccoli@unimib.it

\section{Agnese Infantino}

Università degli Stud di Milano-

Bicocca - Italia

agnese.infantino@unimib.it

\section{Para citar este artigo:}

ZUCCOLI, Franca Giuliana; INFANTINO, Agnese. Curricolo zero-sei: conoscenze fatte di scoperte e riflessioni. Revista Linhas. Florianópolis, v. 19, n. 40, p. 38-53, maio/ago. 2018. 


\section{ECEC curriculum for children aged 0-6 years: knowledge based on discovery and reflection}

\begin{abstract}
In this paper, we examine Law 107/2015 and the subsequent Legislative Decree n.65/2017 with a view to identifying and discussing the educational implications of introducing an "integrated 'birth to six years' early childhood education system", the perspective from which all Italian early childhood services are now called to develop their educational ideas, practices and offerings for 0 - to 6-year-olds. Both the legislative framework and the state of the art in research on childhood point to the need for a holistic approach to development in the early years, and in keeping with this perspective, the proposed 0-6 curriculum should be holistically interpreted and designed, linking art, science, and languages without rigid disciplinary divisions. Hence, we reflect on the specific pathways of discovery and knowledge construction that characterize certain disciplinary areas, arguing that dividing up knowledge by sectors, as an excessive "secondarizaton" of learning has accustomed us to do, is actually very distant from the praxis of researchers and scholars. Ultimately, we recommend proceeding by trial and error, experimenting with divergent pathways to learning, avoiding aiming for a single shared outcome to be forced on all learners alike, and identifying previously implemented learning approaches that may be usefully incorporated into the new early childhood curriculum.
\end{abstract}

Keywords: Early Childhoood Educational Services. Children's Education. Continuity of Education. Art and Science. Discovery and Exploration.
Curriculum

zero-seis:

conhecimentos feitos de descobertas e reflexões

\section{Resumo}

O artigo examina a Lei italiana 107/2015 e o subsequente Decreto Legislativo n. ${ }^{\circ} 65$ de 2017 para analisar e discutir as implicações pedagógicas ligadas ao estabelecimento do "Sistema integrado de educação e instrução desde o nascimento até os seis anos", perspectiva a partir da qual o panorama dos serviços infantis italianos deverão confrontar-se para elaborar ideias, propostas, práticas educacionais relacionadas ao currículo zeroseis. $O$ quadro legislativo e os aprendizados do mundo da pesquisa sobre a infância convergem para esboçar a necessidade de agir a partir de uma abordagem holística ao desenvolvimento nos primeiros anos de vida e de acordo com essa visão, incluindo a proposta de um currículo zero-seis, pede para ser interpretada e explicitada de forma holística, conectando arte, ciência, linguagens além de qualquer distinção disciplinar. As reflexões propostas por este artigo examinam então as especificidades dos percursos de descoberta e de conhecimento próprios de algumas disciplinas, mostrando como a divisão setorial, à qual uma "secundarização" excessiva de aprendizagem nos acostumou, é um elemento extremamente distante de prática desses mesmos pesquisadores e estudiosos. O proceder por tentativas, experimentando percursos dissonantes também, longe da busca de um único resultado, forçosamente idêntico para todos, é, portanto, uma modalidade sugerida aqui, recuperando e valorizando experiências realizadas, ao longo dos anos, exatamente nos currículos destinados às crianças.

Palavras-chave: Serviços Educacionais e Escolares para a Infância. Continuidade Educativa. Arte. Ciência. Descoberta. Exploração. Currículo. 


\section{Lo zero-sei nella Legge 107: nuove sfide pedagogico-organizzative per i} servizi

Nella tradizione della pedagogia dell'infanzia italiana l'educazione dei bambini è da sempre stata tematizzata nella prospettiva dello zero-sei, in particolare nell'ottica della continuità tra servizi, nello specifico il nido d'infanzia e la scuola dell'infanzia, rivolti rispettivamente ai bambini fino ai tre anni, il primo, e dai tre ai sei anni, la seconda. La prospettiva zero-sei, declinata in termini di continuità, più che corrispondere quindi a un'effettiva organizzazione dei servizi sul campo ha riguardato soprattutto l'orientamento pedagogico espresso nelle progettualità e nelle pratiche educative di chi nei servizi e nella ricerca si occupa dell'educazione negli anni dell'infanzia. Ora invece la Legge 107 del 2015 e il successivo Decreto Legislativo n. 65 del 2017 assumono a tutti gli effetti la prospettiva zero-sei come criterio di organizzazione dei servizi educativi e della scuola dell'infanzia nell'ambito del "Sistema integrato di educazione e istruzione dalla nascita ai sei anni", istituito appunto con la nuova legge. In questo contributo saranno prese in esame le più importanti implicazioni poste dal quadro normativo e le aperture interpretative sullo zero-sei che possono essere praticate nelle proposte educative rivolte ai bambini nell’ambito dei servizi educativi e della scuola dell'infanzia.

II nuovo quadro legislativo definisce novità importanti che chiedono di essere interpretate e tradotte nei fatti nel corso del tempo modificando e creando nuove logiche nel lavoro educativo, realizzando al meglio le possibilità offerte dalla legge e, allo stesso tempo, facendo i conti con i chiaroscuri che, come in tutte le leggi, sono presenti anche nella Legge 107 e nel relativo Decreto attuativo n.65.

Ma quali sono queste importanti novità? La prima riguarda indubbiamente l'art. 1 del Decreto n. 65 che fissa i principi e le finalità del Sistema integrato di educazione e istruzione da zero a sei anni: "1. Alle bambine e ai bambini, dalla nascita fino ai sei anni, per sviluppare potenzialità di relazione, autonomia, creatività, apprendimento in un adeguato contesto affettivo, ludico e cognitivo, sono garantite pari opportunità di educazione e di istruzione, di cura, di relazione e di gioco, superando disuguaglianze e barriere territoriali, economiche, etniche e culturali. 2. Per le finalità di cui al comma 1 viene progressivamente istituito in relazione all'effettiva disponibilità di risorse finanziarie, umane e strumentali, il 
Sistema integrato di educazione e di istruzione per le bambine e i bambini in età compresa dalla nascita fino ai sei anni" Già nelle parole di questi primi commi del Decreto n.65 si pongono con chiarezza alcuni temi di fondo che segnalano tutta la complessità del Sistema integrato zero-sei nel quale via via andranno a convergere e fondersi elementi di culture dell'infanzia espresse dal nido e dalla scuola dell'infanzia che ad oggi hanno forti differenziazioni e identità ben distinte. È forse questa la ragione per cui nel testo del Decreto pare essere necessario individuare e nominare espressamente sia le polarità che riguardano più da vicino la cultura educativa del nido (cura, relazione, educazione) sia quelle più riconducibili alla tradizione culturale della scuola dell'infanzia (istruzione, gioco, ... ) connotando il Sistema sia sul versante dell'educazione sia su quello dell'istruzione. In molti, tanto dall'interno del nido quanto dalla scuola dell'infanzia, con l'emanazione del Decreto n. 65, hanno espresso forti preoccupazioni di fronte all'istituzione del Sistema integrato zero-sei in difesa di quelli che ritengono capisaldi delle culture specifiche del nido e della scuola dell'infanzia che, in una formula estremamente sintetica e riduttiva, potremmo individuare come cultura della "cura" al nido e degli "apprendimenti" alla scuola dell'infanzia. I timori reciproci riguardano cioè da un lato la percezione che la cultura della scuola dell'infanzia possa circoscrivere la centralità della relazione di cura che il nido percepisce spesso come tratto specifico dell'educazione proposta nei primi anni di vita e, viceversa, chi opera nell'ambito della scuola dell'infanzia sente minacciata la propria identità dalla cultura del nido intravvedendo il rischio di un eccessivo investimento proprio sulle relazioni di cura a scapito di quelle più direttamente orientate sul versante degli apprendimenti e della costruzione dei saperi. Queste reazioni, reciprocamente giocate in posizioni di difesa, esprimono molto chiaramente l'attuale contesto in cui le due realtà, quella del nido e della scuola dell'infanzia, hanno progressivamente alimentato le proprie specificità pedagogiche e interpretato le pratiche di continuità educativa a partire dall'idea dell'esistenza di due mondi distinti, indubbiamente aperti alla comunicazione e al dialogo, ma pur sempre differenziati e distinti. E questa distinzione, che in alcune situazioni si traduce in vera e propria distanza nonostante a volte i servizi condividano spazi comuni, si fonda su idee e rappresentazioni dei bambini nei loro primi sei anni di vita fortemente segmentate in zero-tre e tre sei, 
operando una scissione netta e radicale in una fase della vita che vive però solo nei confini delle realtà e delle istituzioni e non corrisponde di fatto né alle esperienze della vita reale dei bambini - che normalmente convivono nel quotidiano con bambini di altre età - né nelle acquisizioni del mondo della ricerca che sempre più chiaramente, pur ribadendo la densità dei processi di costruzione dell'identità nei primi due anni di vita, pongono in modo inequivocabile l'accento su processi che si snodano all'insegna della continuità e dell'integrazione in una visione olistica dello sviluppo del bambino. Oggi questa nuova legge registra quindi una buona sintonia con la visione di bambino proposta dalla ricerca psico-pedagogica ma non è del tutto in sintonia con la cultura dell'educazione e con le pratiche diffuse ed espresse sul campo al nido e alla scuola dell'infanzia rispetto alle quali tratteggia un orizzonte nuovo, ponendo obiettivi a lungo termine, che richiedono una progressiva costruzione di condizioni concrete per la loro realizzazione. In altre parole siamo in una situazione politica e culturale molto diversa dall'epoca dell'approvazione della Legge 1044 del 1971 istitutiva del nido che, al contrario, registrava le spinte culturali espresse con forza dal basso, non solo dal mondo dei servizi ma da una mobilitazione sociale diffusa nel mondo sindacale, politico, femminista. Oggi la Legge 107 per certi versi piove dall'alto rispetto al mondo degli educatori e degli insegnanti e non può che incontrare inevitabili chiusure e resistenze ponendo all'orizzonte una realtà, quella dello zero-sei, per la quale dall'interno dei servizi, tranne isolate realtà, non esistono allo stato attuale chiare sensibilità e attenzioni mature. In questo senso lo zero-sei sarà una sfida e una prospettiva pedagogica e culturale tutta da costruire nel tempo, ponendosi come evoluzione degli equilibri e degli assetti pedagogico-organizzativi attuali e richiedendo col tempo la capacità di immaginare altri modi e altri assetti pedagogico-organizzativi per i servizi educativi rivolti ai bambini nei primi sei anni di vita. Certo va detto che la complessità dell'attuazione di un Sistema integrato è dovuta anche in buona parte al fatto che nel corso degli anni si è andato definendo un sistema di gestione articolato e plurale in cui, al fianco dell'ente pubblico, si sono sempre più affermati nella gestione dei servizi anche altri soggetti privati e del privato sociale e confessionale e che perciò esiste un'oggettiva complessità di ordine organizzativo e amministrativo percepibile concretamente quando ad esempio, in uno stesso territorio sono presenti un nido 
comunale affidato a una cooperativa sociale, una scuola dell'infanzia gestita direttamente dallo stato e una realtà privata confessionale che gestisce nido e scuola dell'infanzia. Come si può porre la questione di un curricolo zero-sei e di una rappresentazione condivisa di bambino da zero a sei anni in situazioni come questa in cui esistono diverse figure di coordinamento, gruppi di lavoro distinti e separati che non sperimentano mai occasioni sistematiche e continuative di lavoro in comune? Si tratterà di individuare modi e forme per porre in continuità e dialogo fattivo e regolare soggetti molto diversi tra loro, espressione di culture educative orientate a valori e tradizioni gestionali molto diverse, che ad esempio fanno anche riferimento a inquadramenti contrattuali per i lavoratori spesso assai diversi tra loro. Quella di porre in continuità realtà così diverse per costituire un Sistema integrato di educazione e istruzione non è indubbiamente un'operazione scontata e di immediata realizzazione. Questo è del resto un altro passaggio esplicito del Decreto n. 65 che prende atto della pluralità esistente e la esprime sottolineando proprio il valore della continuità nello specifico al comma 3 dell'articolo 1: "Il sistema integrato di educazione e istruzione: promuove la continuità del percorso educativo e scolastico, con particolare riferimento al primo ciclo di istruzione, sostenendo lo sviluppo delle bambine e dei bambini in un processo unitario, in cui le diverse articolazioni del Sistema integrato di educazione e istruzione collaborano attraverso attività di progettazione, di coordinamento e di formazione comuni". In altre parole questo comma del decreto per creare le reali condizioni di unitarietà e continuità di un percorso integrato parrebbe puntare, anche in questo caso, su una prospettiva di lungo periodo, tutta da costruire, in cui la garanzia di organicità e sistematicità viene assegnata alle funzioni di coordinamento e alla condivisione di percorsi formativi in servizio, leve strategiche per porre in atto il cambiamento auspicato dalla legge. Il compito di creare le premesse di fatto per un sistema integrato parrebbe dunque essere affidato da un lato al coordinamento unitario e dall'altro alla cultura professionale che si potrà condividere nell'ambito di percorsi di formazione in servizio comuni tra personale del nido e personale della scuola dell'infanzia. Saranno quindi strategiche le funzioni di coordinamento, vero asse per garantire tenuta e coerenza al sistema, e le funzioni svolte dalla formazione come luogo deputato alla promozione di idee, pensieri, cultura e pratiche professionali non giocate astrattamente 
ma profondamente contestualizzate e connesse con la realtà dei servizi. Implicitamente si lanciano alcune indicazioni operative di non poco conto che tuttavia non sarà immediato e semplice rendere effettive in una traduzione operativa. L'elemento che, infatti, rende non agevole l'attuazione di una visione organica e sistemica tra nido e scuola dell'infanzia risiede nel fatto che la formazione di base resta distinta in due specifici percorsi universitari uno, quello in Scienze dell'Educazione con indirizzo specifico per infanzia, della durata di tre anni, rivolto agli educatori del nido e l'altro, quello in Scienze della Formazione Primaria, della durata di cinque anni, rivolto agli insegnanti. Sarà una bella sfida avvicinare e connettere con la formazione in servizio e con la progettazione condivisa figure professionali che provengono da percorsi di formazione di base assai diversi tra loro, non solo per durata. Certamente è importante aver affidato al Ministero dell'istruzione, dell'università e della ricerca le funzioni di indirizzo, coordinamento e promozione del Sistema integrato su tutto il territorio nazionale, includendo così i servizi per la prima infanzia che finora erano stati gestiti da diversi ministeri. Non basta comunque compiere operazioni strutturali seppure così rilevanti per promuovere una cultura educativa organica e coerente in grado di confrontarsi con l'individuazione di un curricolo zero-sei che implica una visione esplicita delle occasioni e proposte formative e di crescita pensate nei primi sei anni di vita.

\section{Per un curricolo zero-sei}

Riflettere sul curricolo non significa tanto selezionare informazioni e conoscenze da proporre ai bambini, nella logica di stabilire un programma di contenuti di apprendimento, quanto piuttosto di interrogarsi sui processi di crescita dei bambini e sulla coerenza delle offerte all'interno dei servizi. Con quali elementi della cultura in senso ampio è importante che un bambino nelle diverse età entri in contatto per trarne effettivo beneficio per il suo sviluppo? Quali esperienze è opportuno che un bambino compia per acquisire competenze e abilità contestualizzate e coerenti per comprendere, ma anche cambiare e interpretare in modo innovativo il mondo culturale di cui è parte? La definizione di un curricolo pone la necessità di adottare una logica centrata sul bambino nell'arco dei suoi primi sei anni di vita indagando le modalità con cui anche in forme 
spontanee e autonome i bambini entrano in contatto con il mondo naturale, sociale e relazionale, e se ne appropriano per costruire saperi e conoscenze in forme non sempre del tutto prevedibili da parte degli adulti. Posto in questi termini il curricolo zero-sei interroga la nostra visione di bambino e ci impone di adeguare le nostre proposte a un'interpretazione olistica dello sviluppo che non scinde mente-corpo nelle esperienze umane e soprattutto in quelle dei bambini che, in forma molto più evidente degli adulti, vivono questa profonda connessione senza operare scindendo la dimensione affettiva da quella cognitiva o relazionale. Da questo punto di vista, i punti d'arrivo della ricerca sulle prime età della vita sono oggi riferimenti essenziali per comprendere e interpretare le esperienze di crescita e formazione nella prima infanzia mediante modelli che superano definitivamente la contrapposizione tra mente-corpo, cultura-ambiente, affetticognizione a vantaggio di approcci centrati sul valore dell'intersoggettività assunta come sistema motivazionale di base e come "condizione di umanità" (Stern, 2004, pag. 81). I bambini fin dalla nascita manifestano, infatti, una spiccata sensibilità e propensione per il coinvolgimento relazionale con l'adulto o gli adulti che si prendono cura di loro, esprimendo un bisogno umano fondamentale e innato di intersoggettività intesa come relazione di intimità psicologica intensa e profonda. È nella trama di queste dinamiche, che coinvolgono profondamente giorno dopo giorno il piccolo e i suoi caregiver, che si plasma la struttura identitaria profonda dell'individuo mentre contribuisce a orientare e dare forma alla natura delle interazioni interpersonali cui il bambino stesso partecipa come partener attivo a tutti gli effetti. Questo reciproco intreccio di sguardi compone la storia di crescita e formazione di ogni essere umano, infatti come ci ricorda Stern "abbiamo bisogno di incontrare lo sguardo dei nostri simili per formarci come individui $e$ mantenerci tali" (Stern, 2004, pag. 89) La categoria della reciprocità, piuttosto che non la logica deterministica e dicotomica causa-effetto, ci aiuta a comprendere processi fondamentali dello sviluppo umano che, per compiersi, necessitano profondamente dell'incontro intersoggettivo con gli altri esseri umani dal momento che "è solo quando veniamo riconosciuti da un'altra persona che riceviamo la conferma di essere noi stessi" (Reddy, 2008 pag. 96) Concepito in questi termini, lo sviluppo dei bambini fin dalle più tenere età comporta l'attivazione organica di processi che sono ad un tempo emotivi, 
cognitivi, relazionali, sociali nell'ambito di dinamiche in cui è sempre centrale la natura interattiva di ogni esperienza. Molto chiaramente Reddy descrive questi processi segnalando come "il bambino impara il significato da come gli adulti rispondono alla manifestazione dei suoi stati emotivi" (Reddy, 2008, pag. 95) tanto che "il significato non deriva astrattamente dall'uso di categorie ma dall'interazione in atto, ha una natura interattiva" (Reddy, 2008, pag. 92) Le prime fasi della vita sono essenziali per sperimentare e apprendere nell'interazione con le altre persone capacità di attenzione, sintonizzazione affettiva, condivisione e imitazione che perdureranno per tutta la vita come condizione costante della possibilità di apprendere e modificare i nostri modelli di comprensione e interazione con la realtà. Queste consapevolezze trovano ulteriore conferma anche dal mondo della ricerca nell'ambito delle neuroscienze che ci restituiscono l'immagine di una vita neurale intensa fin dalla nascita grazie all'attività del nostro cervello che, dotato di grande plasticità cellulare, nasce per la relazione e si modella nel corso della nostra vita, ma soprattutto entro i primi due anni, nell'intreccio tra predisposizione genetica e interazioni con la complessità dell'ambiente e delle relazioni umane. Come afferma Stern la nostra vita mentale è frutto di co-costruzione con gli altri. Se assumiamo questo quadro, la riflessione sul curricolo per i bambini da zero a seri anni può essere condotta in una nuova luce che restituisce centralità al bambino e alle dinamiche intrapersonali e interpersonali di co-costruzione della conoscenza. Risulta del tutto fuorviante immaginare di isolare condizioni di apprendimento in cui proporre dall'esterno ai bambini una serie di contenuti con cui confrontarsi e di cui impossessarsi come se la conoscenza procedesse per progressiva assimilazione e non fosse al contrario un atto insopprimibilmente creativo. I bambini, con le loro esplorazioni spontanee e con le scoperte autonome, segnalano che arte, scienze, linguaggi nei primi anni di vita sono vissuti come complesso organico olisticamente connesso con le emozioni, i ricordi, le idee, le ipotesi che via via trovano elaborazione nell'esperienza intersoggettiva con gli adulti che abitano il mondo dei piccoli. Bambini e adulti insieme nei contesti educativi partecipano a processi di ricerca continui ed è in questa prospettiva che diventa prolifico interrogarsi sul curricolo da zero a sei anni. 


\section{Quale idea di esperienze significative e di costruzione condivisa delle conoscenze?}

In questa seconda parte del contributo, a partire da quanto evidenziato nelle pagine precedenti e in piena adesione con quanto lì affermato, si vuole riflettere in modo puntuale su quali potrebbero essere le modalità di proposta relativamente alle azioni e ai contenuti da realizzare per e con i bambini. Questo passaggio è pensato senza voler mai scindere la dimensione delle relazioni di cura, sentita come più specifica dal nido, con quella rivolta all'acquisizione di apprendimenti, vista come propria dalla scuola dell'infanzia, anzi continuamente mischiandole, contaminandole, affermando che nessuna azione educativa può in alcun modo privarsi di uno di questi aspetti, rischiando se no di risultare inevitabilmente monca. Questi suggerimenti devono, infatti, tener conto imprescindibilmente delle esperienze significative realizzate negli anni passati, come pure in quelli attuali, sia nei servizi educativi zero-tre, sia nelle scuole dell'infanzia tre-sei, legandole in modo sostanziale alle Indicazioni Nazionali per il Curricolo emanate nel 2012. Si vuole chiarire come questa connessione alle Indicazioni, non sia qui intesa nella funzione di un'applicazione pedissequa, in un certo qual senso volendo scolarizzare in modo anticipato anche il segmento zero-tre, scelta che sarebbe realmente scellerata, ma sia pensata nell'ottica della attivazione di un movimento di pensiero che salvaguardi le differenze, valorizzando le continuità e le discontinuità. Non si tratta ovviamente di un'idea sistematizzata e codificata, ma solo di un primo ragionamento, che dovrebbe essere agito in primo luogo da quanti lavorano nelle due istituzioni e che può rimettere in azione nuovi pensieri dal punto di vista della valorizzazione di quanto compiuto in molte situazioni: osservando, agendo, documentando, riflettendo, valutando sia il proprio operato, sia i passaggi, le soste, i progressi realizzati dai bambini. L'idea è dunque quella di un'attenzione verso un bambino o meglio dei bambini unici, con percorsi di crescita fluidi, aperti, interrogativi che si modificano, si fermano e poi proseguono, che dovrebbero incontrare nel loro cammino ambienti educativi, costruiti con attenzione, supportati da pensieri adulti che favoriscono l'incontro con l'altro e gli altri, che permettono la sperimentazione e la costruzione di conoscenze al contempo personali e condivise. Qui allora un primo passo è quello che ci porta a riflettere su quali siano i punti 
che potremmo pensare come imprescindibili all'interno delle esperienze significative che adulti, intesi come educatori e insegnanti, propongono ai bambini all'interno di contesti formativi, nell'ottica di un percorso che mira a incrementare e a sistematizzare, in modo condiviso: le osservazioni, le scoperte, le riflessioni, come pure i passaggi, gli ostacoli, gli snodi.

\section{A partire dalle Indicazioni Nazionali 2012}

Come si è detto nel precedente paragrafo le Indicazioni Nazionali possono essere utilizzate come un primo spunto, non tanto per rendere più scolarizzato il segmento zerotre anni, ma al contrario per trarre da questo scritto quelle riflessioni che possono essere condivise volendo costruire come adulti ambienti educativi significativi e rifiutandosi di fornire un sapere parcellizzato, precostituito suddiviso in microsettori disciplinari. Leggendo con attenzione il testo, infatti, si trovano numerosi passaggi in cui si chiede di riflettere su una visione del sapere diversa da quella molto spesso proposta dalla scuola, invitando i docenti a fare un passaggio di consapevolezza. Si tratta di suggerimenti che valgono come monito anche e soprattutto per gli ordini successivi, scuola primaria, seidieci anni, e scuola secondaria di secondo grado, undici-quattordici, ma che posti come premessa ci fanno capire l'intento degli estensori. Ecco dunque trascritto qui solo un passaggio, individuato a titolo emblematico tra i molti, inserito per comprendere il disegno sottostante a queste Indicazioni che per la prima volta in Italia cercano di promuovere un pensiero complessivo su educazione e insegnamento/apprendimento, pensando a bambini e ragazzi in termini globali e non frammentati, in un percorso che parte dai tre e arriva fino ai quattordici anni: "Fin dalla scuola dell'infanzia, nella scuola primaria e nella scuola secondaria di primo grado l'attività didattica è orientata alla qualità dell'apprendimento di ciascun alunno e non ad una sequenza lineare, e necessariamente incompleta, di contenuti disciplinari. I docenti, in stretta collaborazione, promuovono attività significative nelle quali gli strumenti e i metodi caratteristici delle discipline si confrontano e si intrecciano tra loro, evitando trattazioni di argomenti distanti dall'esperienza e frammentati in nozioni da memorizzare." (Indicazioni, 2012, p.17) L'invito è dunque quello, in qualsiasi ordine, a mantenere un rapporto dinamico, globale e olistico 
con le discipline, in cui si evidenzi un'attenzione mirata a non separare, rendendo sterili, le singole informazioni e i soli concetti. Proprio per rendere praticabile questo percorso si suggerisce di denominare queste occasioni campi d'esperienza, ecco cosa viene evidenziato in termini di azioni dell’adulto: “I campi di esperienza. Gli insegnanti accolgono, valorizzano ed estendono le curiosità, le esplorazioni, le proposte dei bambini e creano occasioni di apprendimento per favorire l'organizzazione di ciò che i bambini vanno scoprendo. L'esperienza diretta, il gioco, il procedere per tentativi ed errori, permettono al bambino, opportunamente guidato, di approfondire e sistematizzare gli apprendimenti. Ogni campo di esperienza offre un insieme di oggetti, situazioni, immagini e linguaggi, riferiti ai sistemi simbolici della nostra cultura, capaci di evocare, stimolare, accompagnare apprendimenti progressivamente più sicuri." (Indicazioni, 2012, p.24) Se dunque questo scritto, che si rivolge anche ai percorsi successivi e che rimane un importante passaggio a livello nazionale, poiché ha decretato la necessità di un pensiero complessivo e condiviso tra i diversi insegnanti ed educatori che operano nei vari ordini di un percorso scolastico obbligatorio, afferma a gran voce tra i punti ritenuti imprescindibili quelli legati alla non frammentarietà del sapere e alla sperimentazione diretta, allora possiamo tranquillamente confermare che questa posizione può e deve essere necessariamente sostenuta anche con bambini molto più piccoli in un cammino che su questi punti non dovrebbe incontrare fratture.

\section{Pasticciamenti, sguardi, sperimentazioni e scoperte}

A partire da quanto sostenuto dalle Indicazioni Nazionali, che ci parlano di esperienza diretta e di gioco, proviamo a procedere nell'ambito di quello che potrebbe sembrare a una prima lettura un passaggio legato solo all'educazione scientifica, ma che in realtà può rientrare a pieno titolo nel campo più ampio di un'esplorazione non solo razionale, ma anche corporea (su questo punto va sottolineata la necessità di percorsi educativi che non dimentichi mai il corpo), che coinvolge tutti gli ambiti del sapere. È interessante utilizzare qui il termine "pasticciamento" citando l'inventore di questa modalità operativa, il fisico David Hawkins che nella sua attività d'insegnamento aveva notato come molti studenti delle superiori e dell'università incontrassero costanti 
difficoltà nel momento in cui oltre a ricordare delle nozioni di fisica memorizzate, dovevano trasferire queste competenze ad altro. La sua intuizione era stata quella di capire che questi ragazzi e giovani uomini non avevano sufficientemente giocato da piccoli con i vari materiali, vivendo una mancanza di sperimentazione pratica dapprima nel contesto familiare e successivamente nei primi anni della loro educazione. Questo fatto impediva loro di comprendere realmente quando andavano studiando, per ovviare a ciò secondo Hawkins bisognava mettere in campo differenti strategie d'insegnamentoapprendimento. "Il non aver sufficientemente toccato, pasticciato cose e oggetti, il non avere giocato con elementi naturali e artificiali, l'aver avuto poco tempo per realizzare piccole scoperte, per individuare ipotesi da confermare o confutare, influisce pesantemente come elemento negativo nella successiva formazione." (Zuccoli, 2010, p.107) Per questo motivo aveva iniziato a proporre una modalità di manipolazione esplorativa aperta, che così descrive: "Un tempo molto più lungo di quello comunemente consentito dovrebbe essere dedicato a un lavoro esplorativo - chiamatelo gioco se volete, ma io lo chiamo lavoro - libero e non guidato. Ai bambini verranno dati materiali ed equipaggiamenti - cose - ed essi avranno il permesso di costruire, provare, sondare e sperimentare senza che vi si sovrappongano domande e istruzioni. Questa fase la chiamo fase del Pasticciamento [...]." (Hawkins, 1979, p. 85). A questa fase seguivano poi momenti successivi legati alla riflessione metacognitiva su quanto osservato, al dibattito, alla lezione conferenza e alla sistematizzazione (queste ultimi passaggi più teorici erano pensati per ragazzi più grandi). Ritornando al pasticciamento Hawkins dopo poche pagine ci ricorda ancora come sia fondamentale non perdere questa modalità all'interno di ogni luogo educativo: "Questa fase è importante, soprattutto, perché porta nella scuola la fonte di quasi tutto quello che i bambini hanno già imparato, le radici del loro sviluppo morale, intellettuale ed estetico. [...]. Quando restringiamo lo spazio dell'educazione a quella che avviene nelle scuole, buttiamo via, a nostro rischio, il metodo di quel primo, spettacoloso progresso." (Hawkins, 1979, pp. 87-88). Con il passaggio del tempo questa fase di lavoro evolve, anche se si mantiene non più con caratteristiche infantili, ma strettamente legata a quell'aspetto dell'infanzia che lui chiama "il tipo di sondaggio autodisciplinato ed esplorativo che è l'essenza della creatività." (Hawkins, 1979, p.88) Sempre proseguendo in questo filone che ci parla di 
esplorazioni e scoperte, possiamo accostarci a un'altra lettura che pone in relazione il pensiero dei bambini con le modalità di azione e di scoperta di Charles Darwin, individuate grazie allo studio scrupoloso dei suoi Taccuini giovanili (Pievani, Sala \& Serrelli, 2011). Nessuna pretesa in questo paragone proposto di cedere al rischio di valorizzare oltre misura il procedere infantile, ma solo la voglia esplicitata dagli autori di indagare una dimensione epistemologica propria della scoperta e di capire, tra gli altri aspetti, cosa usare proficuamente come educatori e in quale maniera. L'idea diviene, dunque, quella di svelare almeno in parte cosa può avvenire nel processo di scoperta, allontanando l'immagine romantica di un attimo solitario e inconsapevole, una sorta di miracolo, proponendo invece un processo collettivo e partecipativo, con la propria comunità di riferimento -scienziati o alunni che siano- che si alimenta di analogie, metafore, esperimenti, un cantiere aperto insomma, una sorta di bricolage di idee. (Pievani, Sala \& Serrelli, 2011, p.5, p.20). Alcuni passaggi che ci vengono suggeriti da utilizzare con i bambini sono quelli, tra gli altri: della messa in relazione, della pertinenza, del riferimento all'autorità (intesa come necessità di confronto e legame con altre fonti: educatori, adulti o pari), dell'esperimento, dell'immagine analogica, del modello, delle ipotesi alternative. (Pievani, Sala \& Serrelli, 2011, pp.59-60) Se a partire dalla scoperta in un ambito di matrice prettamente scientifica passiamo a osservare quanto ha compiuto l'arte, in particolare in campo contemporaneo, anche se sul contemporaneo andrebbero effettuati dei distinguo e delle precisazioni (Didi-Huberman, 2007; Sgarbi, 2012), possiamo trovare pure qui la presenza di materiali eterogenei che raccontano di una ricerca inesauribile nel campo della specificità materica, iniziando con l'accostamento e arrivando alla reinvenzione. "Per questa strada assumono statuto artistico i materiali bruti e gli oggetti quotidiani, l'opera diventa una miscela variabile di caso e progetto, nascono generi nuovi come l'operaambiente, o a metà strada tra pittura e scultura" (Gallo, 2011, p. 15). Anche se, come ci ricorda Gillo Dorfles, in ogni epoca: “L'opera d'arte, inoltre, risulta strettamente legata al suo medium - al suo mezzo espressivo - condizionata dai materiali in cui è realizzata, inseparabile dunque dalla stessa costituzione materiale e formale." (2004, p. 15) Un altro passaggio significativo, non necessariamente l'ultimo o posto in questo ordine, risulta allora quello legato a un'attenzione oltre che alla sperimentazione, alla "percezione" 
intesa complessivamente non limitandosi allo sguardo, affiancata da un'azione educativa che proponga un tempo dedicato ad accrescere queste sensibilità. Come ci ricorda Caterina Zaira Laskaris, in Grammatiche della percezione, è importante insistere, anche con i bambini, sulla "[...] consistenza unitaria di questo tempo dinamico e doppio, composto da due momenti che si devono fondere in un atteggiamento globale di comunicazione con il mondo e di conoscenza di esso- includendo però all'interno anche noi stessi, al contempo soggetti e destinatari del vedere- [...]. Mostrare attenzione per la percezione non come attuazione di un godimento solipsistico del fatto artistico, ma come momento esso stesso creativo e vitale per il nostro rapporto con ciò che ci circonda e con ciò che siamo (come sentiamo, percepiamo, vediamo le cose e che cosa esse ci dicono), comporta il recupero di uno spessore profondo, che mette in gioco le categorie di tempo e spazio e quelle di espressione e giudizio." (2017, p.36)

Queste riflessioni qui riportate, che hanno spaziato dai campi più propriamente scientifici, arrivando a indagare, seppur velocemente, i domini dell'arte, sono state qui proposte per rendere valore a quanto in molti luoghi dell'educazione già si realizza, quando si permette ai bambini di misurarsi con materiali e allestimenti differenti, ricordandoci tra le altre cose l'invito di Elinor Goldschmied con il suo Cestino dei tesori (1996), e si sostiene l'esplorazione con domande, azioni, riproposizioni di altri materiali, documentando con attenzione i passaggi compiuti dai bambini. Questa rapida incursione in alcune discipline voleva solo confermare come tra nido e scuola dell'infanzia, salvaguardando le specifiche differenze l'alleanza tutta da fondare sia su una visione di cultura e di costruzione condivisa della conoscenza che abbia uno sguardo olistico e non settoriale. Questo passaggio fondamentale fatto di esplicitazione, messa in comune a partire dai servizi necessita di un tempo significativo e sperimentale per distillare le esperienze precedenti e proporle agli altri, costruendo così, anche se a piccoli passi, un Sistema realmente Integrato. 


\section{Riferimenti}

Didi-Huberman Georges, "Costruire la durata” in Jean-Luc Nancy, Georges Didi-Huberman, Nathalie Heinich, \& Jean-Christophe Bailly, Del contemporaneo. Saggi su arte e tempo, Bruno Mondadori, Milano, 2007, pp.21-52.

Dorfles Gillo, Le oscillazioni del gusto. L'arte d'oggi tra tecnocrazia e consumismo, Skira, Milano, 2004.

Gallo Francesca, "Tecniche e materiali nuovi nelle avanguardie storiche", in Silvia Bordini (a cura di), Arte contemporanea e tecniche. Materiali, procedimenti, sperimentazioni, Carocci, Roma, 2011, pp. 15-36.

Goldschmied Elinor, Jackson Sonia, Persone da zero a tre anni: crescere e lavorare nell’ambiente del nido, Edizioni Junior, Bergamo, 1996 (1994).

Hawkins David, Imparare a vedere. Saggi sull'apprendimento e sulla natura umana, Loescher, Torino, 1979 (1974).

Laskaris Caterina Zaira, Grammatiche della percezione, Mimesis Edizioni, Milano-Udine, 2017. Pievani Telmo, Sala Marcello \& Serrelli Emanuele, La scoperta tra scienziati e bambini. Il caso dei Taccuini giovanili di Charles Darwin, Edizioni ETS, Pisa, 2011.

Reddy Vasudevi, Cosa passa per la testa di un bambino. Emozioni e scoperta della mente, Raffaelo Cortina Editore, Milano 2010 (2008)

Sgarbi Vittorio, L'arte è contemporanea ovvero l'arte di vedere l'arte, Bompiani, Milano, 2012.

Stern Daniel N., Il momento presente, Raffaello Cortina Editore, Milano 2005 (2004)

Zuccoli Franca, Dalle tasche dei bambini... Gli oggetti, le storie e la didattica, Ed. JuniorSpaggiari, Parma 2010.

Universidade do Estado de Santa Catarina - UDESC

Programa de Pós-Graduação em Educação - PPGE

Revista Linhas

Volume 19 - Número 40 - Ano 2018 revistalinhas@gmail.com 